\title{
An Association Study of the Matrix Metalloproteinase-7, -26 Genetic Polymorphisms on the Risk of Ischemic Stroke
}

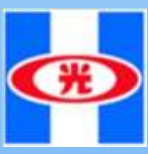

Li-Ming Lien ${ }^{1,2}$, Meng-Tien Tsai ${ }^{3}$, Fang-I Hsieh ${ }^{3}$, Hung-Yi Chiou ${ }^{3}$

${ }^{1}$ Department of Neurology, Shin-Kong WHS Memorial Hospital, Taipei, Taiwan

${ }^{2}$ School of Medicine, College of Medicine, Taipei Medical University, Taipei, Taiwan

${ }^{3}$ School of Public Health, College of Public Health, Taipei Medical University, Taipei, Taiwan

Background: Previous studies indicated that matrix metalloproteinase-7 (MMP-7) and MMP-26 were associated with plaque stability. Therefore, we aimed to study the association of genetic polymorphisms of MMP-7, -26 with ischemic stroke.

Methods: A total of 1000 study subjects (500 ischemic stroke patients and 500 age-, sex- matched healthy controls) were recruited in our study. Information of demographic data, lifestyle, and disease history were collected from the study subjects using a structured questionnaire. The genotypes of selected SNPs from MMP-7 (rs10502001, rs11568818) and MMP-26 (rs2499953, rs2499966) were determined by polymerase chain reaction-restriction fragment length polymorphism (PCR-RFLP). Multivariate logistic regression models were used to analyze the relationship between genetic polymorphisms and risk of ischemic stroke.

Results: After adjusting age, gender, education, obesity, smoke, alcohol, hypertension, diabetes and heart diseases, a protective effect for ischemic stroke was found in study subjects with MMP-26 rs2499966 A allele (OR=0.633, 95\% C.I. $=0.461-0.868)$. There was a 23.7\% decreased risk of developing ischemic stroke for each additional A allele ( $\mathrm{p}$ for trend $=0.039$ ). In study subjects with obesity, a 3.5 -fold $(95 \%$ C.I. $=1.840-6.596)$ increased odds for ischemic stroke were observed in MMP-7 rs10502001 T allele carriers. However, this was not seen in the subjects without obesity.

Conclusion: MMP-26 rs2499966 A allele was protective against ischemic stroke. However, a higher risk for ischemic stroke was seen in MMP-7 rs10502001 T allele carriers in obesity subgroup. Therefore, subjects who carry MMP-7 rs10502001 T allele have to avoid becoming obesity.

Table 1. The distribution of demographic information, disease history, and life style among ischemic stroke cases and controls

\begin{tabular}{lccc}
\hline \hline Variables & Cases & Controls & P value \\
& $\mathrm{N}=500$ & $\mathrm{~N}=500$ & \\
\hline Age $( \pm \mathrm{SD})$ & $61.33 \pm 11.07$ & $61.17 \pm 10.99$ & 0.8085 \\
Feamls & $167(33.40 \%)$ & $167(33.40 \%)$ & 1.0000 \\
Education & & & \\
$\quad$ Elementary school & $277(56.76 \%)$ & $105(21.65 \%)$ & $<\mathbf{0 . 0 0 0 1}$ \\
$\quad$ Junior/Senior high school & $171(35.04 \%)$ & $184(37.94 \%)$ & \\
$\quad$ University & $40(8.20 \%)$ & $196(40.41 \%)$ & \\
Smoking & $241(48.59 \%)$ & $154(30.86 \%)$ & $<\mathbf{0 . 0 0 0 1}$ \\
Alcohol drinking & $105(21.13 \%)$ & $67(13.40 \%)$ & $\mathbf{0 . 0 0 1 2}$ \\
Hypertension & $374(74.80 \%)$ & $263(52.60 \%)$ & $<\mathbf{0 . 0 0 0 1}$ \\
Diabetes & $227(45.40 \%)$ & $83(17.26 \%)$ & $<\mathbf{0 . 0 0 0 1}$ \\
Heart disease & $123(24.65 \%)$ & $78(15.92 \%)$ & $\mathbf{0 . 0 0 0 6}$ \\
Hyperlipidemia & $414(82.97 \%)$ & $405(81.00 \%)$ & 0.4195 \\
BMI $\left(\mathrm{kg} / \mathrm{m}^{2}\right)$ & $25.34 \pm 3.63$ & $24.6 \pm 3.12$ & $\mathbf{0 . 0 0 0 6}$ \\
\hline \hline
\end{tabular}

Chi-square test for categorical variables and Student's $\mathrm{t}$ test for continuous variable

Table 3. Association between MMP 7 rs10502001 and risk of ischemic stroke after stratifying by smoking, alcohol drinking and obesity

\begin{tabular}{lllccl}
\hline \hline Variables & & Genotype & $\begin{array}{c}\text { Cases }(\%) \\
\mathrm{N}=500\end{array}$ & $\begin{array}{c}\text { Controls }(\%) \\
\mathrm{N}=500\end{array}$ & $\mathrm{OR}^{\mathrm{a}}(95 \% \mathrm{C} . \mathrm{I})$. \\
\hline Smoking & No & CC & $131(51.57)$ & $175(50.72)$ & 1.000 \\
& & $\mathrm{CT}+\mathrm{TT}$ & $123(48.43)$ & $170(49.28)$ & $1.040(0.681-1.587)$ \\
& Yes & CC & $127(52.92)$ & $85(55.19)$ & 1.000 \\
& & CT+TT & $113(47.08)$ & $69(44.81)$ & $1.137(0.701-1.845)$ \\
Alcohol & \multirow{2}{*}{ No } & CC & $205(52.43)$ & $227(52.42)$ & 1.000 \\
drinking & & CT+TT & $186(47.57)$ & $206(47.58)$ & $1.152(0.814-1.630)$ \\
& Yes & CC & $54(51.92)$ & $34(50.75)$ & 1.000 \\
& & CT+TT & $50(48.08)$ & $33(49.25)$ & $0.894(0.418-1.915)$ \\
Obesity & No & CC & $187(55.33)$ & $194(48.99)$ & 1.000 \\
& & CT+TT & $151(44.67)$ & $202(51.01)$ & $0.761(0.523-1.106)$ \\
& Yes & CC & $64(44.76)$ & $66(66.67)$ & 1.000 \\
& & CT+TT & $79(55.24)$ & $33(33.33)$ & $3.484(1.840-6.596)^{* * * *}$ \\
\hline \hline
\end{tabular}

a: adjusted by age, sex, education, obesity, smoking, alcohol drinking, hypertension, diabetes, heart disease $* * p<0.005, * * * p<0.0001$
Table 2. The distribution of genetic polymorphisms of MMP 7 and MMP 26 among patients with ischemic stroke and controls

\begin{tabular}{|c|c|c|c|}
\hline SNPs & $\begin{array}{c}\text { Cases }(\%) \\
\mathrm{N}=500\end{array}$ & $\begin{array}{c}\text { Controls (\%) } \\
\mathrm{N}=500\end{array}$ & $\mathrm{OR}^{\mathrm{a}}(95 \%$ C.I. $)$ \\
\hline \multicolumn{4}{|c|}{$\begin{array}{l}\text { MMP 7 } \\
\text { rs10502001 }\end{array}$} \\
\hline $\mathrm{CC}$ & $262(52.61)$ & $261(52.20)$ & 1.000 \\
\hline $\mathrm{CT}$ & $204(40.96)$ & $197(39.40)$ & $1.269(0.911-1.769)$ \\
\hline TT & $32(6.43)$ & $42(8.40)$ & $0.643(0.355-1.165)$ \\
\hline \multicolumn{3}{|c|}{ Dominant model } & $1.126(0.823-1.539)$ \\
\hline \multicolumn{3}{|c|}{ Additive model } & $0.976(0.765-1.245)$ \\
\hline \multicolumn{4}{|c|}{$\begin{array}{l}\text { MMP 7 } \\
\text { rs11568818 }\end{array}$} \\
\hline AA & $422(84.74)$ & $428(85.60)$ & 1.000 \\
\hline AG & $73(14.66)$ & $68(13.60)$ & $1.038(0.659-1.635)$ \\
\hline GG & $3(0.60)$ & $4(0.80)$ & $0.576(0.095-3.507)$ \\
\hline \multicolumn{3}{|c|}{ Dominant model } & $1.007(0.646-1.570)$ \\
\hline \multicolumn{3}{|c|}{ Additive model } & $0.977(0.647-1.475)$ \\
\hline \multicolumn{4}{|c|}{$\begin{array}{l}\text { MMP 26 } \\
\text { rs2499953 }\end{array}$} \\
\hline AA & $240(48.19)$ & $253(50.60)$ & 1.000 \\
\hline AG & $212(42.57)$ & $206(41.20)$ & $0.958(0.691-1.328)$ \\
\hline GG & $46(9.24)$ & $41(8.20)$ & $0.955(0.542-1.683)$ \\
\hline \multicolumn{3}{|c|}{ Dominant model } & $0.957(0.701-1.307)$ \\
\hline \multicolumn{3}{|c|}{ Additive model } & $0.969(0.762-1.233)$ \\
\hline
\end{tabular}

\section{MMP 26}

rs2499966

CC $291(58.32) \quad 273(54.6) \quad 1.000$ reference

CA $173(34.67) \quad 201(40.2) \quad 0.590(0.424-0.820) *$

AA $\quad 35(7.01) \quad 26(5.2) \quad 0.992(0.509-1.935)$

Dominant model $0.633(0.461-0.868) *$

Additive model $\quad 0.763(0.590-0.986) *$

a: adjusted for age, sex, education, obesity, smoking, alcohol drinking, hypertension, diabetes, heart disease

The $4^{\text {th }}$ European Stroke Organization Conference, May,16-18, 2018, Gothenburg, Sweden. 